journal club

\title{
Inzidenz und Prävalenz psychiatrischer Komorbiditäten bei MS-Patienten
}

Fragestellung: Besteht eine höhere Inzidenz und Prävalenz von psychiatrischen Erkrankungen bei MS-Patienten?

Hintergrund: Die höhere Prävalenz von psychiatrischen Erkrankungen bei MS-Patienten werden die meisten Neurologen und Nervenärzte sicher auch ohne größere epidemiologische Studien bestätigen können. Einige kleinere epidemiologische Studien konnten eine erhöhte Prävalenz zwar aufzeigen, ohne allerdings die jeweiligen Erkrankungen in ihrer Breite und im Detail zu differenzieren. In dieser Studie ging es nicht nur um den Einfluss verschiedener Komorbiditäten auf die Mortalität von MS-Patienten, sondern speziell um die klinische Prävalenz der verschiedenen psychiatrischen Erkrankungen wie Depression, Angststörungen, bipolaren Erkrankungen und anderen Psychosen.

Patienten und Methoden: Die Datensätze von kanadischen Gesundheitsbehörden aus vier Provinzen von insgesamt 44.452 MS-Patienten wurden mit den Daten von 220.849 alters-, geschlechts- und regionskorrigierten Kontrollen verglichen.

Marrie RA, Fisk JD, Tremlett $\mathrm{H}$ et al. Differences in the burden of psychiatric comorbidity in MS vs the general population. Neurology 2015; 85: 1972-9
Ergebnisse: Der Anteil von Frauen unter den MS-Patienten war erwartungsgemäß mit $71 \%$ höher als unter Männern. In der Gruppe der MS-Patienten lag die jährliche Inzidenz von Depressi- onen bei 979 (oder 0,98\%), von Angststörungen bei 638, von bipolaren Erkrankungen bei 328 und von Schizophrenie bei 60 Fällen pro 100.000 Einwohnern. Damit lag die Inzidenz der Depression bei MS-Patienten bei knapp $1 \%$ der Gruppe, in der Kontrollgruppe hingegen bei $0,72 \%$, während die Prävalenz bei $20,1 \%$ in der MS-Gruppe jedoch nur bei 11,9\% in der Kontrollgruppe lag. Interessanterweise war die Prävalenz der Depression in der MS-Gruppe bei Frauen um etwa $25 \%$ höher als bei Männern. In der Kontrollgruppe betrug der Unterschied jedoch $50 \%$, sodass insgesamt in der MS-Gruppe im Verhältnis mehr Männer unter einer Depression litten als in der Kontrollgruppe. Die Inzidenz der Angststörungen lag in der MS-Gruppe bei $0,63 \%$, in der Kontrollgruppe bei $0,42 \%$, wobei die Prävalenz von Angststörungen in der MS-Gruppe 8,7\% und in der Kontrollgruppe 5,1 \% betrug. Auch hier waren Inzidenz und Prävalenz jeweils bei Frauen höher als bei Männern. Die Inzidenz bipolarer Störungen lag in der MS-Gruppe bei 0,33\%, die Prävalenz bei $4,7 \%$, während in der Kontrollgruppe die Inzidenz bei $0,16 \%$ und die Prävalenz bei 2,3 \% lag. Bipolare Störungen waren etwas häufiger bei Männern als bei Frauen. Die Inzidenz der Schizophrenie betrug in der MS-Gruppe 0,06\%, die Prävalenz $1,28 \%$. In der Kontrollgruppe ließ sich die Inzidenz aus statistischen Gründen nicht genau errechnen, die Prävalenz lag jedoch bei 1,03\% und damit unter der Prävalenz in der MS-Gruppe.

Schlussfolgerungen: Die Studie bestätigt die hohe Inzidenz und Prävalenz psychiatrischer Komorbiditäten bei MS-Patienten; Depressionen und Angsterkrankungen stehen im Vordergrund.

\section{- Kommentar von Volker Limmroth, Köln}

\section{Prävalenz psychiatrischer Erkrankungen bei MS-Patienten nicht trivial}

Man kann die kanadischen Kollegen nur beglückwünschen, dass es ihnen möglich ist, Studien dieser Art realisieren zu können, was aufgrund der Datenschutzparanoia in Deutschland leider kaum möglich ist. Die Hauptbotschaft der Studie ist sicherlich nicht komplett neu und bestätigt die hohe Komorbidität von psychiatrischen Erkrankungen bei MS-Patienten. Aber die Güte der Studie liegt in der Reichhaltigkeit des Datenmaterials und der detaillierten Analyse vieler Untergruppen. Wichtigste Komorbidität ist die Depression - danach muss davon ausgegangen werden, das rund $20 \%$ aller MS-Patienten unter einer Depression leiden, Patientinnen sogar bis zu $26 \%$, wobei auch Männer stärker betroffen sind als in der Normalbevölkerung. Zweitwichtigste psychiatrische Komorbidität ist die Angststörung bei knapp $9 \%$ der Patienten, auch hier deutlich häufiger bei Frauen. Bipolare Störungen sind mit knapp 5\% seltener und Schizophrenien sind mit 1,3\% zwar knapp häufiger als in der Normalbevölkerung, aber vergleichs- weise selten. Leider liefert auch diese Studie keine Daten zum Einfluss der modernen Immuntherapien auf die Komorbiditäten. Aber völlig eindeutig bleibt: Bei einem knappen Drittel der MS-Patienten, also bei knapp jedem dritten Patienten, muss mit einer psychiatrischen Begleiterkrankung gerechnet werden, wovon die meisten behandlungsbedürftig sind.

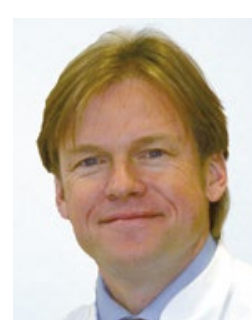

Prof. Dr. med. Volker Limmroth, Köln-Merheim

Chefarzt der Klinik für Neurologie und Palliativmedizin Köln-Merheim E-Mail: LimmrothV@kliniken-koeln.de 